\title{
Analysis of the $A^{1} \Pi$ State on the Basis of the Douglas-Herzberg Bands System in the $\mathrm{CH}^{+}$Ion Molecule
}

\author{
R. Hakalla*, R. KȨPA, W. Szajna And M. ZaChWieja \\ Atomic and Molecular Physics Laboratory, Institute of Physics \\ University of Rzeszów, Rejtana 16a, 35-310 Rzeszów, Poland \\ (Received December 28, 2006; revised version April 27, 2007)
}

This paper presents an attempt of examining the irregularities appearing in a complicated $A^{1} \Pi$ state of the $\mathrm{CH}^{+}$molecule with their reasons provided. By using the experimental data for the $A^{1} \Pi-X^{1} \Sigma^{+}$bands system of the ${ }^{12} \mathrm{CH}^{+}$ion radical, it was proved that the vibrational and rotational quanta of the upper state reveal the same unusual behaviour, i.e. very clear nonlinear dependence on vibrational quantum number $\left(v^{\prime} \geq 3\right)$ of the upper state. Therefore, upper vibrational levels $\left(v^{\prime} \geq 3\right)$ of the $A^{1} \Pi$ state cannot be determined by means of the equilibrium constants calculated in the previous works. Due to so far unidentified $A^{1} \Pi$ state perturbations, the reduction of the wave numbers to the rovibronic parameters was carried out by means of individual, band-by-band analysis method, using with this end in view the nonlinear least squares method introduced by Curl and Dane, and Watson. This method allowed one to make already calculated constants of the rovibronic structure of regular lower state $X^{1} \Sigma^{+}$of $A-X$ system independent of possible perturbations appearing in the upper state of $A^{1} \Pi$ of this system. It also enabled one to calculate for the first time the real (perturbed) term values for the $A^{1} \Pi\left(v^{\prime}=0,1,2\right.$, and 3$)$ state of the ${ }^{12} \mathrm{CH}^{+}$ion molecule. These values suggest that rotational irregularities in the $A^{1} \Pi$ state examined are negligibly small. In order to confirm the nonexistence of rotational perturbations in the $A^{1} \Pi\left(v^{\prime}=0,1,2\right.$, and 3$)$ state, up to the observed $J_{\max }$ level, appropriate graphs of functions $f_{x}(J)$ and $g_{x}(J)$ introduced by Gerö and Kovács, where $x=\mathrm{Q}, \mathrm{PR}$, and $\overline{\mathrm{PR}}$, were drawn. Also, their course was analysed in detail.

PACS numbers: 33.20.Kf

\section{Introduction}

Within the last 60 years, the $\mathrm{CH}^{+}$ion radical has been the subject of almost hundred, both theoretical and experimental, publications as well. This significant amount of papers devoted to this molecule has been inspired by its two important

*corresponding author; e-mail: hakalla@univ.rzeszow.pl 
features:

(a) Its kinetic behaviour is rich in information about many molecule-building reaction paths which take place in the space, as the $\mathrm{CH}^{+}$is a major intermediate of it;

(b) It has only 6 electrons and that is why it is extremely appropriate for $a b$ initio calculations of high accuracy. Comparisons of theory and experiment determine a test, very important for these calculations, which also decides about their quality and correctness.

The beginning of spectroscopic research concerning the $\mathrm{CH}^{+}$radical, goes back to 1941 [1]. At that time, during the Yerkes Observatory Conference, it was suggested that one unidentified group of interstellar absorption lines comes from this particular ion radical. Four individual, very intense lines with 4232.58, 3957.72, and 3745.33 $\AA$ wavelengths, isolated from spectrum reaching our planet from the interstellar space by Dunham [2], Dunham and Adams [3], and Adams [4], seemed to be particularly interesting. This hypothesis has been proved by an analysis of spectrum of the $\mathrm{CH}^{+}$molecule, obtained in laboratory conditions by Douglas and Herzberg [5], who also analysed the $0-0,1-0,2-0$, and $0-1$ bands of the $A-X$ system. Wavelengths of the $R(0)$ line received for the first three bands, occurred to be lines 4232.58, 3957.72, and 3745.33 $\AA$ obtained from the space. It was the first experimentally gained proof that the $\mathrm{CH}^{+}$ion radical is the main element of the interstellar gas clouds. They calculated the vibrational constants for the $A^{1} \Pi$ state $\left(\omega_{\mathrm{e}}^{\prime}=1850.02 ; \omega_{\mathrm{e}} x_{\mathrm{e}}^{\prime}=103.93 \mathrm{~cm}^{-1}\right.$; unfortunately without the experimental uncertainties) for the first time, altogether with some of the equilibrium rotational constants for both the upper $A$ state and the lower $X$ state. These two vibrational constants of the $A$ state fit later discovered higher vibrational levels in a very good manner. Nonetheless, the authors observed that the vibrational levels of the electronic state discussed converge much faster than it had been expected. That is why they drew a false conclusion that in consequence level $v=2$ is the last one and dissociation energy amounts about $5500 \pm 1800 \mathrm{~cm}^{-1}$. The fourth absorption line $(3579.04 \AA)$ observed in the interstellar space seemed to belong to band $3-0$, unobserved in that paper. However, the problem lied in the fact that had it been the truth, the first of all constants, $\omega_{\mathrm{e}} y_{\mathrm{e}}$, would have had an unexpectedly high value, and secondly, three equilibrium vibrational constants would not have fitted a higher vibrational level. Due to these reasons, the authors expressed the opinion that the line $3579.04 \AA$ cannot belong to the $\mathrm{CH}^{+}$molecule. Douglas and Morton [6] verified this conclusion. Thanks to their work it occurred that a relatively high, positive value of vibrational constant $\omega_{\mathrm{e}} y_{\mathrm{e}}$ of the $A^{1} \Pi$ state does exist $\left(\omega_{\mathrm{e}}^{\prime}=1865.35, \omega_{\mathrm{e}} x_{\mathrm{e}}^{\prime}=115.85, \omega_{\mathrm{e}} y_{\mathrm{e}}^{\prime}=2.64 \mathrm{~cm}^{-1}\right)$. Henceforth, the fourth, so far unidentified line from the interstellar space $3579.04 \AA$ also belongs to the $\mathrm{CH}^{+}$radical.

Carré and Doufnay [7] and Carré [8], who examined emission spectra of $\mathrm{CH}_{4}$ and $\mathrm{C}_{2} \mathrm{H}_{2}$ molecules, excited by means of proton impact, observed bands $3-0$ and 
4-0 for the $A^{1} \Pi-X^{1} \Sigma^{+}$system and recorded two new bands system $B^{1} \Delta-A^{1} \Pi$ (0-0, 1-1, and $2-2$ bands) and ${ }^{3} \Sigma-{ }^{3} \Pi(0-0,1-0$, and $2-1)$ in the $\mathrm{CH}^{+}$radical. Unfortunately, they did not provide any conclusion concerning the $A^{1} \Pi$ state. Taking advantage of previous experimental data, Botterud et al. [9] fixed some of molecular parameters for both states of Douglas-Herzberg system. Carrington and Ramsay [10] have analysed eight bands $(0-0,0-1,1-0,1-1,1-2,1-3,2-1$, and $3-1$ ) of the $A^{1} \Pi-X^{1} \Sigma^{+}$system in ${ }^{12} \mathrm{CH}^{+}$. Bands, whose part were levels $v^{\prime \prime}=2$ and $v^{\prime \prime}=3$ of ground state, were observed for the first time. Examining them allowed the authors to calculate more precise bands origin and individual rotational constants for both combining states, altogether with vibrational constants for $X^{1} \Sigma^{+}$state. They also noticed, just like Douglas and Morton [6], clear irregularities having appeared in the $A^{1} \Pi$ state, which made the calculation of equilibrium vibrational and rotational constants for this state impossible. Thus, they have suggested that some ${ }^{3} \Pi$-type state is the reason of the occurrence of these irregularities.

Helm et al. [11] examined some rotationally quasibound levels for different vibrational levels (up to $v^{\prime}=10$ ) of the $A^{1} \Pi$ state of the ${ }^{12} \mathrm{CH}^{+}$ molecule by means of a coaxial fast-ion-beam-laser photofragment spectrometer. It allowed them to estimate the dissociation energy of $A^{1} \Pi$ state to have a value of $D_{0}^{0}=9351(23) \mathrm{cm}^{-1}$. Antić-Jovanović et al. [12] examined the $A-X$ system in the ${ }^{13} \mathrm{CH}^{+}$. The fact that vibrational constants for the $A^{1} \Pi$ $\left(\omega_{\mathrm{e}}^{\prime}=1860.0, \omega_{\mathrm{e}} x_{\mathrm{e}}^{\prime}=115.2\right.$, and $\left.\omega_{\mathrm{e}} y_{\mathrm{e}}^{\prime}=2.6 \mathrm{~cm}^{-1}\right)$ state determined by them also do not fit higher vibrational levels $\left(v^{\prime}>2\right)$ is very interesting. At the same time the constant $\omega_{\mathrm{e}} y_{\mathrm{e}}^{\prime}$ is of a relatively high value. Hoppe et al. [13], among other things, examined the $B^{1} \Delta-A^{1} \Pi$ system in the ${ }^{12} \mathrm{CH}^{+}$molecule by means of chemiluminescence, and reanalysed the $0-0,1-1$, and $2-2$ bands of this system. They determined $T_{\mathrm{e}}^{A^{1} \Pi}=24111 \mathrm{~cm}^{-1}$ and $D_{\mathrm{e}}^{A^{1} \Pi}=10400 \mathrm{~cm}^{-1}$ for this molecule. Next, new electronic transition, namely $C^{1} \Sigma^{+}-A^{1} \Pi$ (0-1 and 1-2 bands) was observed by Macau-Hercot et al. [14].

Lately, Hakalla et al. [15] have received longer than so far observed bands (0-0, 0-1, and 2-1) of the $A-X$ system in the ${ }^{12} \mathrm{CH}^{+}$molecule. On the basis of molecular information obtained from these bands and through the second analysis of bands received by Carrington and Ramsay [10], the following have been calculated: more accurate wave numbers, the improved reduced band system origin $T_{\mathrm{e}}^{A^{1} \Pi}=24118.726(14) \mathrm{cm}^{-1}$, equilibrium molecular constants for the $A^{1} \Pi$ state: $\omega_{\mathrm{e}}^{\prime}=1864.402(22), \omega_{\mathrm{e}} x_{\mathrm{e}}^{\prime}=115.832(14), \omega_{\mathrm{e}} y_{\mathrm{e}}^{\prime}=2.6301(24), B_{\mathrm{e}}^{\prime}=11.88677(72)$, $\alpha_{\mathrm{e}}^{\prime}=0.9163(18), D_{\mathrm{e}}^{\prime}=1.92960(31) \times 10^{-3} \mathrm{~cm}^{-1}$, experimental equilibrium bond lengths of $r_{\mathrm{e}}^{\prime}=1.235053(37)$ and $r_{\mathrm{e}}^{\prime \prime}=1.1308843(30) \AA$ for the $A^{1} \Pi$ and $X^{1} \Sigma^{+}$states, respectively, as well as RKR turning points, and for the first time the addition to the zero-point energy for the both combining electronic states: $Y_{00}^{\prime}=-1.9430 \mathrm{~cm}^{-1}$ and $Y_{00}^{\prime \prime}=1.8953 \mathrm{~cm}^{-1}$. The experimental Franck-Condon factors and $r$-centroids for the system under consideration have also been obtained. 
Forming, disintegrating and abundance of the $\mathrm{CH}^{+}$radical in the space were the subject of very intense research in the last years (see Ref. [16] and references therein). Research into the identification of the $\mathrm{CH}^{+}$spectrum molecule in the interstellar space were also carried out in a large amount (for a review see Refs. $[17,18]$ and references therein). Within the confines of aforementioned works, the first identifications of emission lines of the $\mathrm{CH}^{+}$molecule were made: (i) in comets by Greenstein [19], (ii) outside the Solar System by Balm and Jura [20], (iii) in the Red Rectangle nebula by means of the high resolution spectroscopic observation of the emission lines from the exciting carbon-rich star HD 44179 by Waelkens et al. [21]. Nevertheless, the first identification of absorption lines of the $\mathrm{CH}^{+}$molecule with the interstellar origin was made by Spitzer [22]. Cernicharo et al. [23] were the first ones who recognized the pure rotational spectrum of the $\mathrm{CH}^{+}$in the NGC 7027 planetary nebula. The $\mathrm{CH}^{+}$ion radical is an extremely important factor participating in creation of such important molecules, appearing in the space, as $\mathrm{CH}, \mathrm{C}_{2}, \mathrm{CO}, \mathrm{CN}$, and many others.

In some experimental works mentioned above $[5,6,10]$, it has been observed that the $A^{1} \Pi$ state of the $\mathrm{CH}^{+}$molecule reveals strong irregularities. Experimental vibrational and rotational constants of the $A^{1} \Pi$ state are known for all the levels, from $v=0$ to $v=4$. Unfortunately, the irregularities in the observed vibrational term values of these electronic states make a satisfactory extrapolation of these data, used in order to determine molecular constants of higher vibrational levels impossible. Another problem caused by these disturbances is the fact that no value of $v^{\prime}$ does $\Delta G\left(v^{\prime}\right)=0$. Despite a so big influence of these irregularities on the $A^{1} \Pi$ state structure and spectra connected with the state, their character and source have not been determined yet. The attempt to answer this question has been made within the framework of this project.

\section{The lowest dissociation threshold of the $\mathrm{CH}^{+}$}

Predissociation absorption lines of the $\mathrm{CH}^{+}$for the first time were recorded by Carrington and Sarre [24] by means of an argon ion laser operating in a visible wave band. Cosby et al. [25], and Graff and Moseley [26] analysed them in detail using the visible and near-ultraviolet lines of both argon and krypton ion lasers. Many theoretical ([27] and literature ibidem) and experimental studies ([28, 29] also see references therein) have been conducted concerning the examination of the first dissociation limit and the electronic states combined with them. These works showed that its structure is very specific, i.e., it is governed by the $63.4 \mathrm{~cm}^{-1}$ fine-structure splitting [30] of the $\mathrm{C}^{+}(2 P)$ fragment energy levels [31]. The $A^{1} \Pi$ potential curve, responsible for the radiative transitions to the $\mathrm{CH}^{+}$ground state is adiabatically combined with the higher lying ${ }^{2} P_{1 / 2}$ fine-structure level of $\mathrm{C}^{+}$, while the $X^{1} \Sigma^{+}$potential curve correlates with the lower lying $X^{1} \Sigma^{+}$fine-structure level of $\mathrm{C}^{+}$. That is why it may as well be one of the causes of the irregularities observed in the $A^{1} \Pi$ state. Graph presenting the chart of potential curves for all electronic 


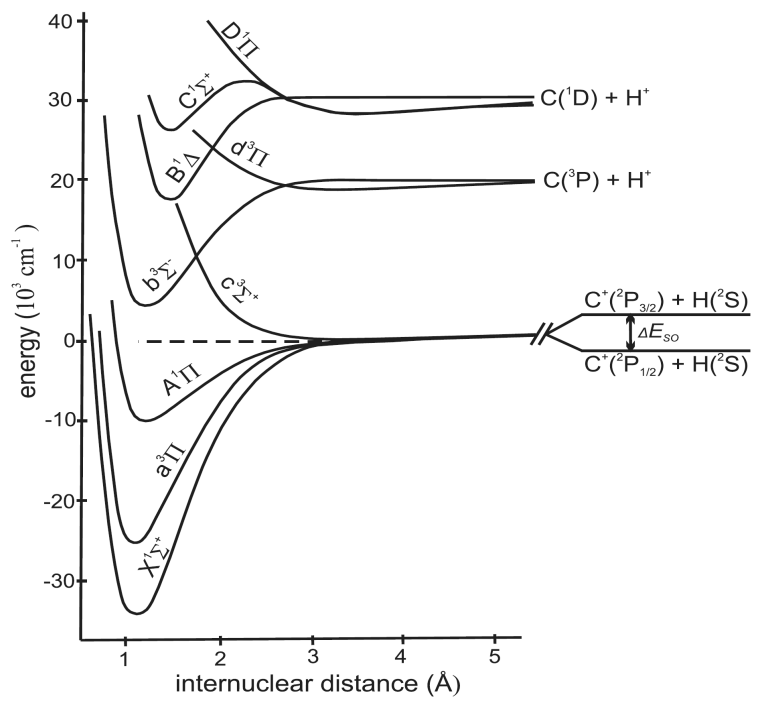

Fig. 1. Potential energy curves for $\mathrm{CH}^{+}$radical correlating to the first three dissotiation limits. Adapted from Ref. [32] and [29]. $\Delta E_{\mathrm{SO}}$ means the spin-orbit splitting of the lowest dissociation threshold (indicated with the broken line). $\Delta E_{\mathrm{SO}}=63.4 \mathrm{~cm}^{-1}$ (after Moore [30]).

states mentioned in the introduction of this paper was shown in Fig. 1 made on the basis of works carried out by Sarre and Whitham [32] and Hechtfischer et al. [29].

\section{Analysis and calculations}

The problem with the $A^{1} \Pi$ state in the $\mathrm{CH}^{+}$molecule is that the equilibrium molecular constants of this state, counted both theoretically and experimentally do not fit its higher levels. This irregularity was observed by Douglas and Herzberg [5] in 1942, next in 1960 by Douglas and Morton [6], and also by Carrington and Ramsay [10] in 1982. So we decided to verify this problem by means of the newest experimental data obtained by Hakalla et al. [15] recently. For this purpose, on the basis of individual values of rovibronic constants obtained in the framework of work [15], and assuming their traditionally recognized polynomial dependence on the vibrational quantum number, by means of calculation based on the weighted least squares method, the equilibrium molecular constants were calculated:

a) vibrational for the $A^{1} \Pi$ and $X^{1} \Sigma^{+}$states, where the $3-1$ band origin was excluded from the calculation (due to the fact that $v^{\prime}=3$ level of the $A^{1} \Pi$ state is suspected of irregularity);

b) rotational for the $A^{1} \Pi$ state, where the $B_{3}^{\prime}$ individual constant was excluded from the calculation owing to the same reasons.

The obtained values are: $T_{\mathrm{e}}^{\prime}=24123.6576(42), \omega_{\mathrm{e}}^{\prime}=1849.2789(60), \omega_{\mathrm{e}} x_{\mathrm{e}}^{\prime}=$ $103.9962(22), B_{\mathrm{e}}^{\prime}=11.89693(56), \alpha_{\mathrm{e}}^{\prime}=0.94654(40) \mathrm{cm}^{-1}$ for the $A^{1} \Pi$ state, as 


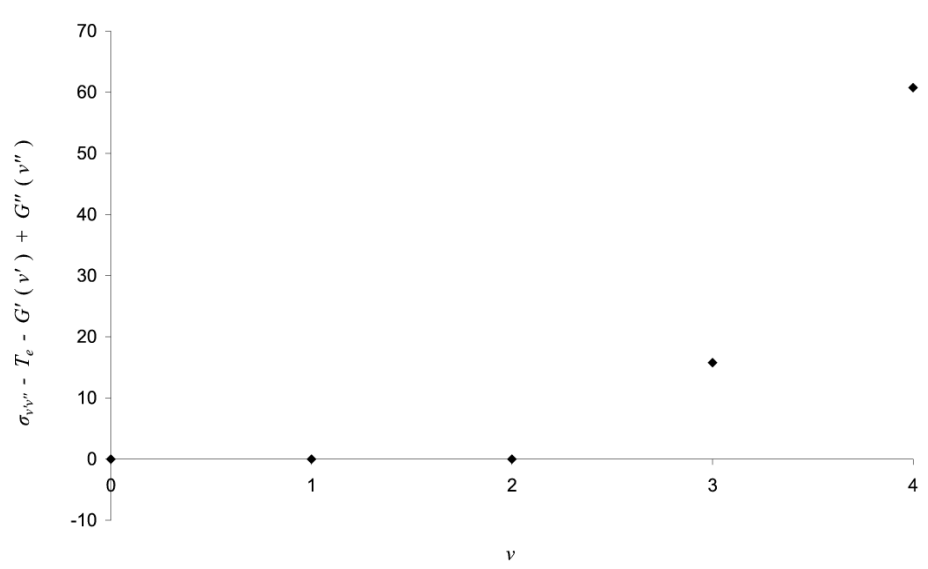

Fig. 2. The deviation of the bands origins of the $v^{\prime}-1$ progression of the $A^{1} \Pi-X^{1} \Sigma^{+}$ system in the ${ }^{12} \mathrm{CH}^{+}$molecule from the position calculated from the formula $\sigma_{v^{\prime}-v^{\prime \prime}}=$ $T_{\mathrm{e}}+G^{\prime}\left(v^{\prime}\right)-G^{\prime \prime}\left(v^{\prime \prime}\right)$. Values of the deviations are given in $\mathrm{cm}^{-1}$. For the $v^{\prime}=0,1$, 2, 3 there are used values of the bands origins given by Hakalla et al. [15] as well as the values of the equilibrium vibrational constants obtained in this paper. For the $v^{\prime}=4$ there are used the value of the $\sigma_{4-1}=26246.6 \mathrm{~cm}^{-1}$ band origin given by Douglas and Morton [6] as well as the values of the equilibrium vibrational constants obtained in this paper.

well as $\omega_{\mathrm{e}}^{\prime \prime}=2857.5609(38), \omega_{\mathrm{e}} x_{\mathrm{e}}^{\prime \prime}=59.3179(11), \omega_{\mathrm{e}} y_{\mathrm{e}}^{\prime \prime}=0.22534^{*} \mathrm{~cm}^{-1}$ for the $X^{1} \Sigma^{+}$state, where ${ }^{*}$ denotes constrained value during the calculations. On their basis we have calculated values of the bands origins of the $v^{\prime}-1$ progression of the $A-X$ system and $B_{v}^{\prime}$ molecular constant of $A^{1} \Pi$ state. Next, their deviations from values counted by means of the following formulae have been portrayed in a chart

$$
\sigma_{v^{\prime}-v^{\prime \prime}}=T_{\mathrm{e}}+G^{\prime}\left(v^{\prime}\right)-G^{\prime \prime}\left(v^{\prime \prime}\right)
$$

for the bands origins (see Fig. 2), and the deviations of $B_{v}$ from the formula

$$
B_{v}=B_{\mathrm{e}}-\alpha_{\mathrm{e}}\left(v+\frac{1}{2}\right)+\gamma_{\mathrm{e}}\left(v+\frac{1}{2}\right)^{2}+\varepsilon_{\mathrm{e}}\left(v+\frac{1}{2}\right)^{3}+\ldots,
$$

which is represented in Fig. 3.

Because of so far unidentified perturbations revealing their presence in the $A^{1} \Pi$ state, there has been a justified suspicion that the reduction of wave numbers of individual bands of $A-X$ system in the $\mathrm{CH}^{+}$molecule by means of nonlinear least squares procedure, in which both analysed states are presented by effective Hamiltonian of Brown et al. [33], provided in many previous publications falsified molecular constants for both upper and lower states. That is why in the present paper it has been decided to verify these calculations and carry out the reduction of wave numbers received by Hakalla et al. [15] and Carrington and Ramsay [10] 


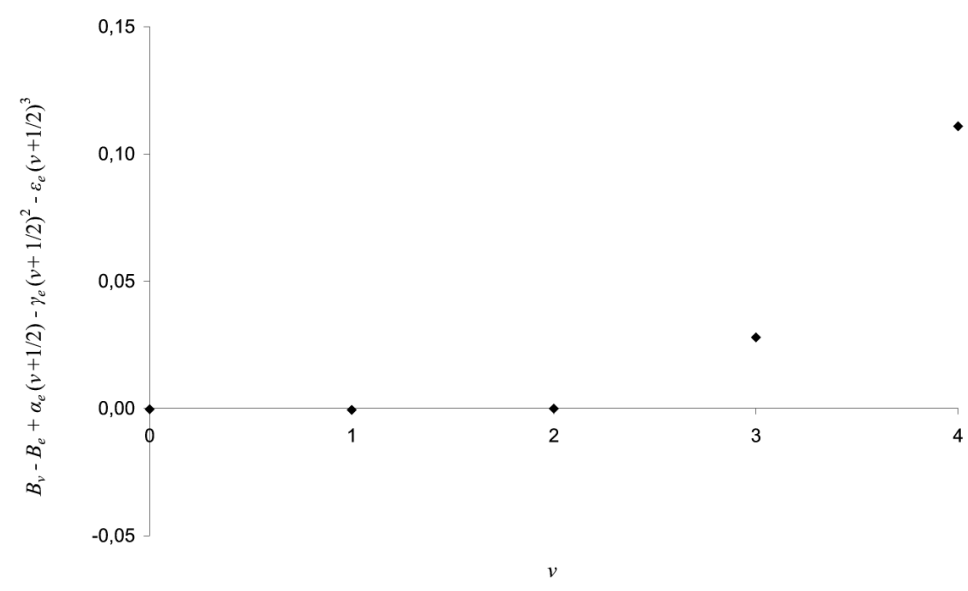

Fig. 3. The deviation of the rotational constants of the $A^{1} \Pi$ state in the ${ }^{12} \mathrm{CH}^{+}$ molecule from the formula $B_{v}=B_{\mathrm{e}}-\alpha_{\mathrm{e}}\left(v+\frac{1}{2}\right)+\gamma_{\mathrm{e}}\left(v+\frac{1}{2}\right)^{2}+\varepsilon_{\mathrm{e}}\left(v+\frac{1}{2}\right)^{3}$. Values of the deviations are given in $\mathrm{cm}^{-1}$. For the $v^{\prime}=0,1,2,3$ there are used values of the rotational constants given by Hakalla et al. [15] as well as the values of the equilibrium rotational constants obtained in this paper. For the $v^{\prime}=4$ there are used the value of the $B_{4}=7.7484 \mathrm{~cm}^{-1}$ band origin given by Douglas and Morton [6] as well as the values of the equilibrium rotational constants obtained in this paper.

to the rovibronic parameters (in the individual band-by-band analysis) by means of the most appropriate method in case of perturbations of one of the states taking place, namely a nonlinear least squares method introduced by Curl and Dane [34] and Watson [35]. Thanks to this method molecular information concerning regular ground state of $X^{1} \Sigma^{+}$, presented in this method by effective Hamiltonian of Brown et al. [33], has been separated from the influence of disturbed $A^{1} \Pi$ state, presented by means of terms. Therefore, constants of the rovibronic structure of the $X^{1} \Sigma^{+}$ state, free from rotational perturbations, as well as the real (perturbed) individual term values for the $v^{\prime}=0,1,2$, and 3 vibrational levels of the irregular $A^{1} \Pi$ state have been received. These terms were merged with regard to $J^{\prime \prime}=0$ of the $v^{\prime \prime}=0$ level of the $X^{1} \Sigma^{+}$state and were shown in Table I. The individual constants of the lower state $X^{1} \Sigma^{+}$, counted by means of this procedure, were compared with appropriate individual constants of this state, obtained by means of reduction of the wave numbers of the $A-X$ system in the ${ }^{12} \mathrm{CH}^{+}$molecule (received by Hakalla et al. [15] and Carrington and Ramsay [10]), to the rotational parameters for the individual bands, by means of the nonlinear least squares procedure, in which both analysed electronic states of this system were presented by effective Hamiltonian of Brown et al. [33]. The effects of this comparison are shown in Table II. 
TABLE I

Merged term values (in $\mathrm{cm}^{-1}$ ) for the $v^{\prime}=0,1,2$, and 3 levels of the $A^{1} \Pi$ state in the ${ }^{12} \mathrm{CH}^{+}$ion radical. Term values for the irregular upper $A^{1} \Pi(v=0,1,2$, and 3) state calculated with regard to $J^{\prime \prime}=0$ of the $v^{\prime \prime}=0$ level of the $X^{1} \Sigma^{+}$ state. Expressions in parentheses represent the uncertainties of the merged values in unit of the last quoted digit, in $\mathrm{cm}^{-1}$.

\begin{tabular}{|c|c|c|c|c|}
\hline \multicolumn{3}{|c|}{$v^{\prime}=0$} & \multicolumn{2}{|c|}{$v^{\prime}=1$} \\
\hline$J$ & $F_{1 e}$ & $F_{1 f}$ & $F_{1 e}$ & $F_{1 f}$ \\
\hline 1 & $23619.7838(30)$ & $23619.6990(39)$ & $25260.1090(25)$ & $25260.0402(27)$ \\
\hline 2 & $23665.5074(33)$ & $23665.2662(41)$ & $25302.0460(29)$ & $25301.8295(32)$ \\
\hline 3 & $23733.9737(40)$ & $23733.5005(47)$ & $25364.8235(45)$ & $25364.3915(42)$ \\
\hline 4 & 23825.0408 (49) & $23824.2589(55)$ & $25448.2799(44)$ & $25447.5783(51)$ \\
\hline 5 & $23938.5169(60)$ & 23937.3609 (64) & $25552.2568(56)$ & $25551.1900(59)$ \\
\hline 6 & $24074.1579(64)$ & $24072.5370(70)$ & $25676.4560(59)$ & $25674.9830(65)$ \\
\hline 7 & $24231.6776(69)$ & $24229.5341(75)$ & $25820.6020(64)$ & $25818.6573(65)$ \\
\hline 8 & $24410.7510(73)$ & $24408.0106(78)$ & $25984.3522(66)$ & $25981.8533(71)$ \\
\hline 9 & $24610.9585(78)$ & $24607.5818(82)$ & $26167.2724(75)$ & $26164.1658(81)$ \\
\hline 10 & $24831.9052(82)$ & $24827.8218(87)$ & $26368.9088(82)$ & $26365.176(12)$ \\
\hline 11 & $25073.0615(87)$ & 25068.267 (10) & $26588.7524(32)$ & $26584.244(38)$ \\
\hline 12 & $25333.984(10)$ & $25328.347(12)$ & $26826.1941(382)$ & $26820.923(81)$ \\
\hline 13 & $25614.004(12)$ & $25607.514(18)$ & $27080.635(81)$ & \\
\hline 14 & 25912.445 (18) & $25905.141(34)$ & $27351.34(14)$ & \\
\hline 15 & 26228.788 (34) & $26220.502(67)$ & $27637.58(21)$ & \\
\hline 16 & $26562.183(64)$ & $26552.91(11)$ & & \\
\hline 17 & $26911.87(11)$ & $26901.61(17)$ & & \\
\hline \multicolumn{3}{|c|}{$v^{\prime}=2$} & \multicolumn{2}{|c|}{$v^{\prime}=3$} \\
\hline$J$ & $F_{1 e}$ & $F_{1 f}$ & $F_{1 e}$ & $F_{1 f}$ \\
\hline 1 & $26692.4653(97)$ & $26692.403(12)$ & $27932.6238(58)$ & $27932.5670(63)$ \\
\hline 2 & $26730.599(10)$ & $26730.406(13)$ & $27967.0808(67)$ & $27966.9024(73)$ \\
\hline 3 & $26787.675(12)$ & $26787.292(14)$ & $28018.6378(79)$ & $28018.2820(86)$ \\
\hline 4 & $26863.546(14)$ & $26862.905(16)$ & $28087.1386(86)$ & $28086.5472(94)$ \\
\hline 5 & $26957.994(17)$ & $26957.019(18)$ & $28172.3665(89)$ & $28171.4872(95)$ \\
\hline 6 & $27070.780(19)$ & $27069.418(20)$ & $28274.0637(95)$ & $28272.8517(96)$ \\
\hline 7 & $27201.550(21)$ & $27199.757(22)$ & $28391.9307(96)$ & $28390.331(11)$ \\
\hline 8 & $27349.982(22)$ & $27347.708(23)$ & $28525.661(11)$ & $28523.619(22)$ \\
\hline 9 & $27515.647(23)$ & $27512.854(24)$ & $28674.714(22)$ & $28672.279(59)$ \\
\hline 10 & $27698.061(24)$ & $27694.702(25)$ & $28838.784(59)$ & \\
\hline 11 & $27896.725(25)$ & $27892.776(26)$ & $29017.40(12)$ & \\
\hline 12 & 28111.060 (38) & $28106.470(28)$ & & \\
\hline 13 & 28340.395 (68) & 28335.167 (38) & & \\
\hline 14 & & 28578.188 (68) & & \\
\hline
\end{tabular}


TABLE II

Individual molecular constants (in $\mathrm{cm}^{-1}$ ) of the $X^{1} \Sigma$ state of the ${ }^{12} \mathrm{CH}^{+}$molecule. Uncertainties in parentheses represent one standard deviation in units of the last quoted digit. The values ordered in sequence one under the other stand: (i) for the constants obtained in this work from the individual band-by-band analysis using the nonlinear least squares method suggested by Curl and Dane [34] and Watson [35], where the regular $X^{1} \Sigma$ lower state was represented by the effective Hamiltonian of Brown et al. [33] and the irregular $A^{1} \Pi$ upper state was represented by terms, (ii) and for the constants calculated by the reducing wave numbers of the $A-X$ system in the ${ }^{12} \mathrm{CH}^{+}$molecule to the rotational parameters for individual bands using the nonlinear least squares procedure, where both analysed states are represented by effective Hamiltonian of Brown et al. [33].

\begin{tabular}{|c|c|c|c|c|}
\hline Band & $B_{v}$ & $D_{v} \times 10^{3}$ & $H_{v} \times 10^{7}$ & $\sigma \times 10^{3 c}$ \\
\hline \multirow[t]{2}{*}{$0-0^{a}$} & $13.93076(33)$ & $1.3782(48)$ & $1.12(20)$ & 4.60 \\
\hline & 13.93059 (18) & $1.3763(17)$ & $1.136(59)$ & 3.86 \\
\hline \multirow[t]{2}{*}{$1-0^{b}$} & $13.93035(50)$ & $1.358(11)$ & $-0.57(70)$ & 5.29 \\
\hline & $13.92939(54)$ & $1.3497(85)$ & $-0.69(49)$ & 5.54 \\
\hline \multirow[t]{2}{*}{$0-1^{a}$} & $13.44103(36)$ & $1.3599(71)$ & $1.75(42)$ & 3.88 \\
\hline & $13.44087(25)$ & $1.3481(34)$ & $0.74(21)$ & 3.98 \\
\hline \multirow[t]{2}{*}{$1-1^{b}$} & $13.44112(48)$ & $1.3314(92)$ & $-0.35(51)$ & 4.16 \\
\hline & $13.44079(53)$ & $1.3300(96)$ & $0.40(51)$ & 5.93 \\
\hline \multirow[t]{2}{*}{$2-1^{a}$} & $13.44179(60)$ & $1.3591(81)$ & 1.25 & 2.60 \\
\hline & $13.44137(42)$ & $1.3576(49)$ & $1.27(20)$ & 5.34 \\
\hline \multirow[t]{2}{*}{$3-1^{b}$} & $13.44323(61)$ & $1.427(24)$ & $9.1(27)$ & 2.82 \\
\hline & $13.44203(68)$ & $1.401(21)$ & $7.4(22)$ & 6.06 \\
\hline \multirow[t]{2}{*}{$1-2^{b}$} & $12.95562(91)$ & $1.296(57)$ & $-1.0(71)$ & 3.33 \\
\hline & $12.95587(56)$ & $1.319(15)$ & $1.6(14)$ & 4.86 \\
\hline \multirow[t]{2}{*}{$1-3^{b}$} & $12.47655(49)$ & $1.3431(75)$ & $3.148^{*}$ & 5.51 \\
\hline & $12.47667(78)$ & $1.350(29)$ & $4.5(31)$ & 5.77 \\
\hline \multicolumn{5}{|c|}{$\begin{array}{l}{ }^{a} \text { Wave numbers of the } A-X \text { system in the } \\
{ }^{12} \mathrm{CH}^{+} \text {molecule used for the calculations were } \\
\text { taken from the work of Hakalla et al. }[15] \text {. } \\
{ }^{b} \text { Wave numbers of the } A-X \text { system in the }{ }^{12} \mathrm{CH}^{+} \\
\text {molecule used for the calculations were taken } \\
\text { from the work by Carrington and Ramsay [10]. }\end{array}$} \\
\hline
\end{tabular}

Classification of irregularities observed in $A^{1} \Pi$ state of $\mathrm{CH}^{+}$radical was carried out by means of functions $f_{x}(J)$ and $g_{x}(J)$, where $x=\mathrm{Q}, \mathrm{PR}$, and $\overline{\mathrm{PR}}$, 
introduced by Gerö [36] and Kovács [37] (p. 287-301):

$$
\begin{aligned}
& f_{\mathrm{Q}}(J)=[Q(J)-Q(J-1)] / 2 J, \\
& f_{\mathrm{PR}}(J)=[P(J+1)-P(J)+R(J-1)-R(J-2)] / 2 J, \\
& f_{\overline{\mathrm{PR}}}(J)=[P(J)-P(J-1)+R(J)-R(J-1)] / 2 J, \\
& g_{\mathrm{Q}}(J)=[(J+1) Q(J-1)-(J-1) Q(J)] / 2, \\
& g_{\mathrm{PR}}(J)=(J+1)[P(J)+R(J-2)]-(J-1)[P(J+1)+R(J-1)] / 4, \\
& g_{\overline{\mathrm{PR}}}(J)=(J+1)[P(J-1)+R(J-1)]-(J-1)[P(J)+R(J)] / 4 .
\end{aligned}
$$

The most important area of applying these functions is discovery and identification of rotational perturbations. If irregularities appear in the $\mathrm{Q}$ branch, formulae (3) and (6) should be applied, irrespective of whether this perturbation affects upper or lower state of the system discussed. If irregularities appear in the $\mathrm{P}$ or $\mathrm{R}$ branches, then formulae (4) and (7) or (5) and (8) should be examined, depending on whether the perturbation is observed in upper or lower state. In this paper we have analysed all of these cases, in order to take into consideration a precise examination of irregularities observed. Selected graphs are shown in Fig. 4 and Fig. 5, for the $f_{x}(J)$ and $g_{x}(J)$ functions, respectively. Graphs $f_{x}(J)$ in $J^{2}$ and $g_{x}(J)$ in $J^{2}\left(J^{2}-1\right)$ functions, linear and regular in the unperturbed intervals, will either rise or fall in the neighbourhood of points of perturbation passing
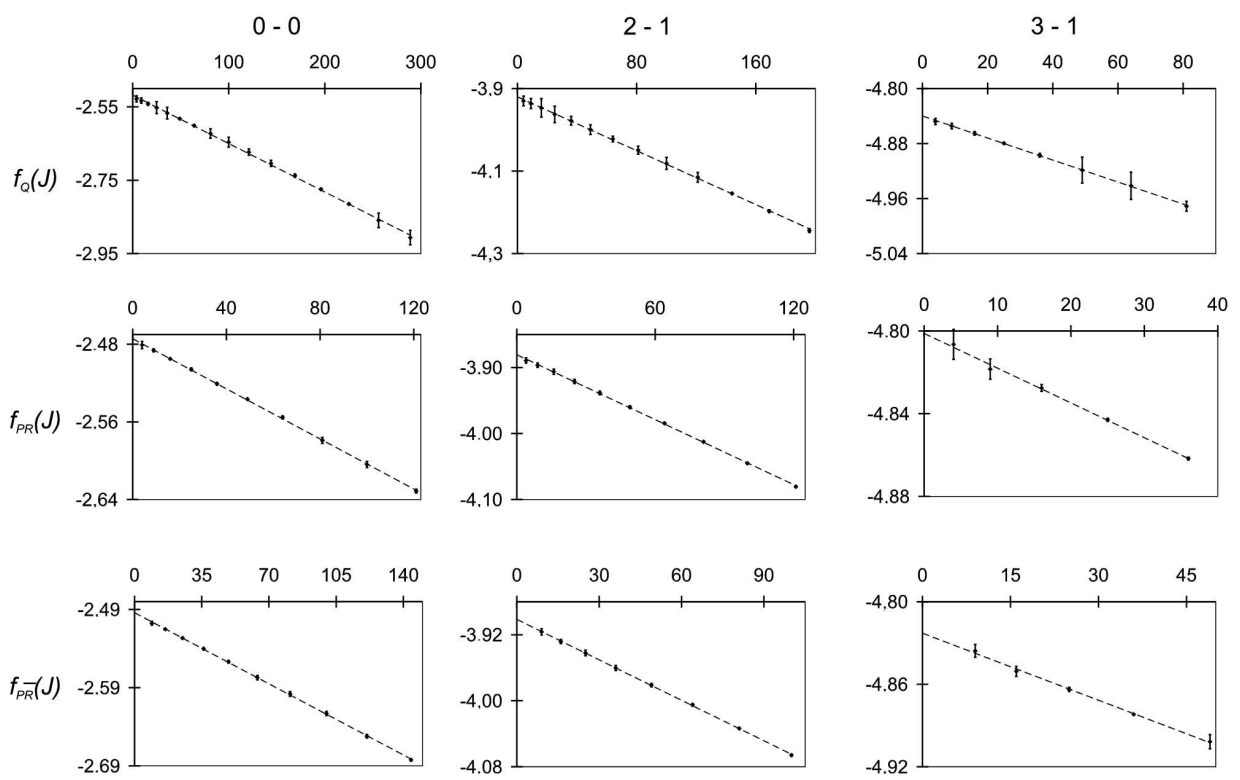

Fig. 4. The $f_{x}(J)$ functions for the $0-0,2-1$, and $3-1$ bands of the $A^{1} \Pi-X^{1} \Sigma^{+}$system in the ${ }^{12} \mathrm{CH}^{+}$molecule. The functions were introduced by Kovács [37], where $x=\mathrm{Q}$, $\mathrm{PR}$, or $\overline{\mathrm{PR}}$; in $\mathrm{cm}^{-1}$ on $y$-axis, and $J^{2}$ values on $x$-axis. 

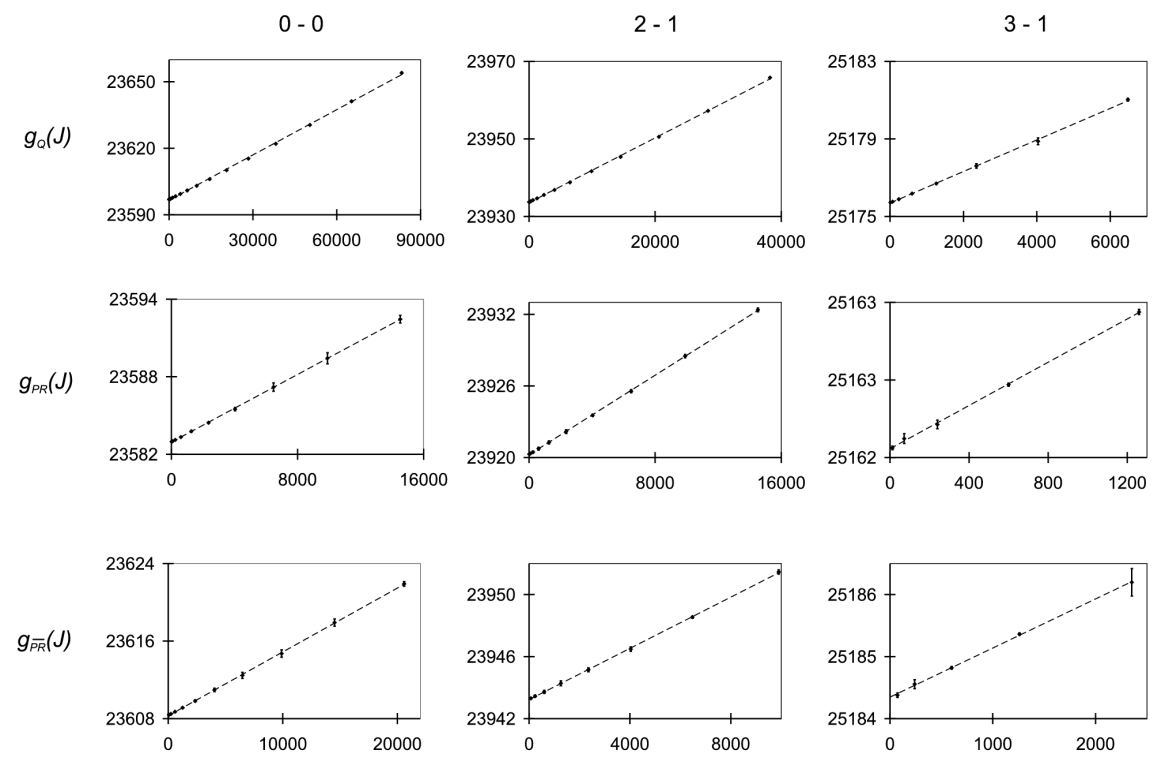

Fig. 5. The $g_{x}(J)$ functions for the $0-0,2-1$, and $3-1$ bands of the $A^{1} \Pi-X^{1} \Sigma^{+}$system in the ${ }^{12} \mathrm{CH}^{+}$molecule. The functions were introduced by Kovács [37], where $x=\mathrm{Q}$, $\mathrm{PR}$, or $\overline{\mathrm{PR}}$; in $\mathrm{cm}^{-1}$ on $y$-axis, and $J^{2}\left(J^{2}-1\right)$ values on $x$-axis.

over into another regular lines, depending on whether the rotational constants of the perturbing term is greater or smaller than that of the perturbed term. These deviations from the linear course of graphs make the fixing of the rotational constants of the perturbing term possible.

\section{Discussion}

So as to examine the irregularities appearing in $A^{1} \Pi$ state of $\mathrm{CH}^{+}$molecule precisely, the equilibrium molecular constants of this state were calculated on the basis of data more accurate than those obtained previously and the latest rovibronic constants of this state received by Hakalla et al. [15] lately. The data connected with $A^{1} \Pi\left(v^{\prime}=3\right)$ level were excluded from this calculation because of the irregularities appearing particularly visibly for the higher levels $\left(v^{\prime}>2\right)$ of this state. On the basis of the equilibrium molecular constants, bands origins of $v^{\prime}-1$ progression in the $A-X$ system of ${ }^{12} \mathrm{CH}^{+}$molecule and rotational constants $B_{v}^{\prime}$, where $v^{\prime}=0,1,2$, and 3 of $A^{1} \Pi$ state have been calculated. This way, the irregularities, counted by means of the constants out of the appropriate values obtained directly from the formula (1) and formula (2), have been charted. The results are shown in Fig. 2 and Fig. 3, respectively. Analysing these graphs, we state the fact that the vibrational and rotational quanta of upper state of $A-X$ state, do reveal the same, unusual behaviour, namely a very vivid nonlinear 
dependence on vibrational quantum number $v^{\prime}$ of the $A^{1} \Pi$ state, and that higher rotational levels $\left(v^{\prime}>2\right)$ of this state cannot be represented within the bounds of experimental uncertainties by the equilibrium constants derived from lower $\left(v^{\prime} \leq\right.$ 2) vibrational levels. Due to this reason, as the further calculations show, the $v^{\prime}=3$ and $v^{\prime}=4$ vibrational levels of the state discussed do not fall in the position which one would predict from the $v^{\prime}=0,1$, and 2 levels, and they converge much more slowly than expected. In fact, if the observed $\Delta G\left(v^{\prime}\right)$ vibrational distances are fitted to a cubic or quartic equation, then for no value of $v^{\prime}$ does $\Delta G\left(v^{\prime}\right)=0$. That is why we concede that fixing the dissociation energy for $A^{1} \Pi$ state in $\mathrm{CH}^{+}$molecule by means of extrapolation of data received for a few first vibrational levels is completely unjustified. However, the dissociation energy $\left(D_{\mathrm{e}}^{A 1}=(14415 \pm 3509) \mathrm{cm}^{-1}\right.$ from Ref. [15]) can be calculated by applying another method: namely if $A-X$ bands system origin and the dissociation energy of the ground state $X$ are known, as well as considering the value of the fine-structure splitting of the lowest dissociation threshold.

Because of the unidentified so far irregularities of $A^{1} \Pi$ state in the $\mathrm{CH}^{+}$ ion radical, the reduction of the wave numbers, received by Hakalla et al. [15] and Carrington and Ramsay [10], to the rovibronic parameters, was conducted by means of the nonlinear least squares method, appropriate for this aim, introduced by Curl and Dane [34] and Watson [35], and applied for an individual band-by-band analysis. Owingly, for the first time the experimental values of the real (perturbed) rotational terms for the irregular electronic $A^{1} \Pi\left(v^{\prime}=0,1,2\right.$, and 3$)$ state have been calculated. The results are presented in Table I. Molecular constants for $X^{1} \Sigma^{+}$state calculated within the framework of this paper, were compared with suitable values received by Hakalla et al. [15] (see Table II). This comparison is extremely surprising since constants of the lower state of $A-X$ system separated from the influence of rotational perturbation originating from higher $A^{1} \Pi$ state are in a significant majority unanimous (within the confines of one standard deviation) with those calculated in Ref. [15]. This conclusion - together with a very good small value of the standard deviations for the calculations carried out with the use of both methods - proves the nonexistence of rotational perturbations of $A^{1} \Pi$ $\left(v^{\prime}=0,1,2\right.$, and 3$)$ state up to observed $J_{\max }$.

Appropriate graphs based on the functions $f_{x}(J)$ and
$g_{x}(J)$, introduced by Gerö [36] and Kovács [37], where $x=\mathrm{Q}, \mathrm{PR}$, and $\overline{\mathrm{PR}}$, were drawn and are presented in Fig. 4 and Fig. 5. Linear, regular course of all these types of graphs prove definitely the fact of nonexistence of rotational disturbances in electronic state $A^{1} \Pi$ for all observed $v^{\prime}=0,1,2$, and 3 vibrational levels (up to the observed $J_{\max }$ ). The exclusion of rotational perturbation in irregular state $A^{1} \Pi\left(v^{\prime}=0,1,2\right.$, and 3$)$, up to the observed $J_{\max }$, of $\mathrm{CH}^{+}$molecule, obviously does not exclude other types of disturbances. Their further identification is open for perspective consideration. 


\section{Conclusions}

This paper presents a modern analysis of complicated $A^{1} \Pi\left(v^{\prime}=0,1,2\right.$, 3 , and 4) state in ${ }^{12} \mathrm{CH}^{+}$molecule as well as an attempt to identify the strong irregularities observed in this electronic state. Undoubtedly, our knowledge about the $\mathrm{CH}^{+}$molecule is still too poor so that the formulation of the final conclusions about the type and origin of these disturbances can not be possible. Nonetheless, the definite exclusion of rotational perturbation (within the framework of observed rotational levels for $v^{\prime}=0,1,2$, and 3 ), together with the first determination of the real (perturbed) values of the rotational terms for $A^{1} \Pi$ state, as well as the calculations of very well determined molecular constants for ground state $X^{1} \Sigma^{+}$, are another very important step towards understanding and recognition of a very interesting $A^{1} \Pi$ state and the character of $\mathrm{CH}^{+}$ion radical.

\section{References}

[1] E.A. Douglas, G. Herzberg, Astrophys. J. 94, 381 (1941).

[2] T. Dunham, Pub. Astron. Soc. Pacific 49, 26 (1937).

[3] T. Dunham, W.S. Adams, Pub. Astron. Soc. Pacific 49, 5 (1937).

[4] W.S. Adams, Astrophys. J. 93, 11 (1941).

[5] E.A. Douglas, G. Herzberg, Canad. J. Res. A 20, 71 (1942).

[6] E.A. Douglas, J.R. Morton, Astrophys. J. 131, 1 (1960).

[7] M. Carré, M. Doufnay, C. R. Acad. Sci. Paris B 266, 1367 (1968).

[8] M. Carré, Physica 41, 63 (1969).

[9] I. Botterud, A. Lofthus, L. Veseth, Phys. Scr. 8, 218 (1973).

[10] A. Carrington, D.A. Ramsay, Phys. Scr. 25, 272 (1982).

[11] H. Helm, P.C. Cosby, M.M. Graff, J.T. Moseley, Phys. Rev. A 25, 304 (1982).

[12] A. Antić-Jovanović, V. Bojović, D.S. Pešić, S. Weniger, Spectrosc. Lett. 16, 11 (1983).

[13] U. Hoppe, A. Kowalski, Ch. Ottinger, Int. J. Mass Spectrom. Ion Processes 127, R1 (1993).

[14] D. Macau-Hercot, F. Rémy, I. Dubois, H. Bredohl, E. Somé, J. Breton, Lab. Astron. High Res. Spectra 2, 319 (1994).

[15] R. Hakalla, R. Kępa, W. Szajna, M. Zachwieja, Eur. Phys. J. D 38, 481 (2006).

[16] T.P. Snow, Astrophys. J. Lett. 402, 73 (1993).

[17] P.J. Sarre, Nature 351, 356 (1991).

[18] L.M. Hobbs, J.A. Thorburn, T. Oka, J. Barentine, T.P. Snow, D.G. York, Astrophys. J. 615, 947 (2004).

[19] J.L. Greenstein, Astrophys. J. 136, 688 (1962).

[20] S.P. Balm, M. Jura, Astron. Astrophys. 261, L25 (1992).

[21] C. Waelkens, H. Van Winckel, N.R. Trams, L.B.F.M. Waters, Astron. Astrophys. 256, L15 (1992). 
[22] L. Spitzer, Physical Processes in the Interstellar Medium, Wiley, New York 1978.

[23] J. Cernicharo, X.-W. Liu, E. Gonzáles-Alfonso, P. Cox, M.J. Barlow, T. Lim, B.M. Swinyard, Astrophys. J. 483, L65 (1997).

[24] A. Carrington, P.J. Sarre, J. Phys. (Paris) 40, C1 (1979).

[25] P.C. Cosby, H. Helm, J.T. Moseley, Astrophys. J. 235, 52 (1980).

[26] M.M. Graff, J.T. Moseley, Chem. Phys. Lett. 83, 97 (1981).

[27] F. Bouakline, T.P. Grozdanov, L. Andric, R. McCarroll, J. Chem. Phys. 122, 044108 (2005).

[28] P.J. Sarre, C.J. Whitham, Chem. Phys. 124, 439 (1988).

[29] U. Hechtfischer, C.J. Williams, M. Lange, J. Linkemann, D. Schwalm, R. Wester, A. Wolf, D. Zajfman, J. Chem. Phys. 117, 8754 (2002).

[30] C.E. Moore, Ionization Potentials and Ionization Limits Derived from the Analysis of Optical Spectra, National Standards Reference Data Series No. 34, NBS, Washington (D.C.) 1970.

[31] U. Hechtfischer, Z. Amitay, P. Forck, M. Lange, J. Linkemann, M. Schmitt, U. Schramm, D. Schwalm, R. Wester, D. Zajfman, A. Wolf, Phys. Rev. Lett. 80, 2809 (1998).

[32] P.J. Sarre, C.J. Whitham, Mol. Phys. 62, 1505 (1987).

[33] J.M. Brown, E.A. Colbourn, J.K.G. Watson, F.D. Wayne, J. Mol. Spectrosc. 74, 294 (1979)

[34] R.F. Curl, C.B. Dane, J. Mol. Spectrosc. 128, 406 (1988).

[35] J.K.G. Watson, J. Mol. Spectrosc. 138, 302 (1989).

[36] L. Gerö, Z. Phys. 93, 669 (1935).

[37] I. Kovács, Rotational Structure in the Spectra of Diatomic Molecules, Akad. Kiadó, Budapest, A. Hilger Ltd., London 1969. 